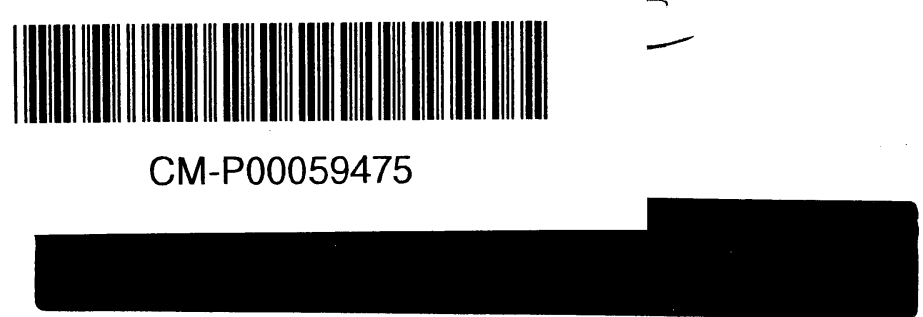

\title{
BEAM LOADING IN RF CAVITIES
}

\author{
R. Garoby
}




\title{
BEAM LOADING IN RF CAVITIES
}

\author{
R. Garoby \\ CERN PS - RF \\ 1211 Geneva 23, Switzerland
}

\section{INTRODUCTION}

Beam loading is the conventional term for the effect of beam passage through an RF cavity installed for the control of some beam parameters such as energy, bunch length, energy spread, etc... The fields developed in such a structure and their effect on the beam can be analyzed as for all other discontinuities of the vacuum chamber. In particular, F. Sacherer's theory of impedances and beam instabilities [1] is perfectly relevant.

However, RF cavities differ largely from the other kind of impedances because they are deliberately tuned at (or near) a frequency where the beam current is large, and because they are equipped with an excitation port for connection to an RF amplifier. For various reasons, a cavity-amplifier system is generally optimized to provide the maximum acceleration per unit of length, with the minimum RF input power. Consequently the cavity impedance is large, and the current from the RF generator is minimized. When the beam current increases, there is then a limit where it becomes non-negligible and a beam loading situation is encountered. The following questions have then to be addressed:

- stability against small perturbations (case of a simple cavity-amplifier set-up, and generalization to the case of a full RF system with servo-loops)

- large transient situations 
- requirements for the RF power amplifier.

Review papers concerning these matters have already been published $[2,3]$. The present one reflects the personal experience of the writer, trying to describe and comment on the current knowledge about these issues, and the available cures.

\section{CONVENTIONS}

\subsection{EQUTVALENT CIRCUIT}

Figure 1 shows the equivalent circuit (Terminology is the one used by F. Pedersen [4]]. The cavity is represented as a simple parallel $R, L, C$, resonator. The amplifier output impedance, transformed to the cavity gap, is included in $\mathrm{R}$ as well as the cavity losses. The beam and the RF amplifier are modelled as ideal current generators.

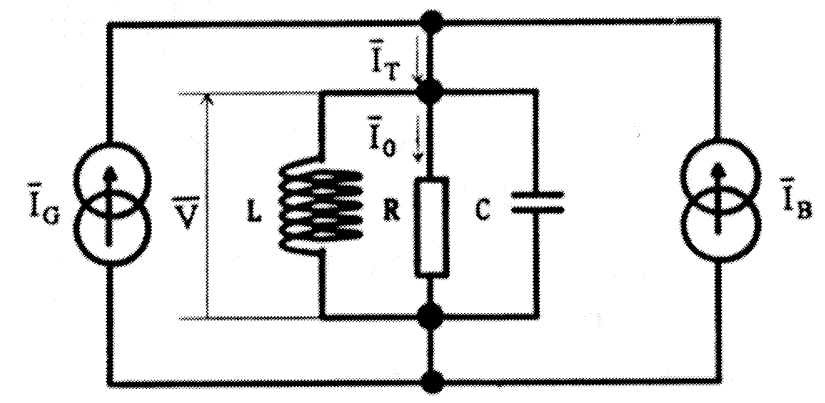

$\overline{\mathrm{V}}$ : voltage across the cavity gap

$\overline{\mathrm{I}}_{G}$ : generator current (transformed to the gap impedance)

$\widetilde{\mathrm{I}}_{\mathrm{B}}$ : beam current

$\overline{\mathrm{I}}_{\mathrm{T}}:$ total current in the cavity

$\overline{\mathrm{I}}_{0}$ : resistive current ( current through $\mathrm{R}$ )

$\mathrm{f}_{\mathrm{R}}$ : cavity resonant frequency

$\mathrm{f}_{C}: R F$ carrier frequency

Figure 1 : Equivalent circuit 


\subsection{STEADY STATE VECTOR DIAGRAM}

Only the RF components (peak values) are considered. This is fully justified if the voltage induced at other frequencies is negligible, either because the currents have negligible spurious frequency components, or because the resonator impedance is very small at these other frequencies. As shown in the vector diagram in figure 2 , the total current $\overline{\mathrm{I}}_{\mathrm{T}}$ flowing through the cavity impedance is the vector sum of the beam and generator currents $\left(\overline{\mathrm{I}}_{\mathrm{B}}\right.$ and $\left.\overline{\mathrm{I}}_{\mathrm{G}}\right) . \overline{\mathrm{V}}$ and $\overline{\mathrm{I}}_{\mathrm{T}}$ are linked by: $\quad \overline{\mathrm{I}}_{\mathrm{T}}=\overline{\mathrm{V}} / \mathrm{Z}_{\mathrm{cav}}=\overline{\mathrm{V}}(1 / \mathrm{R}+\mathrm{jB})$ with $\mathrm{B}=\mathrm{C} \omega-1 /(\mathrm{L} \omega)$. $\vec{I}_{0}=\bar{V} / R$ is the minimum current needed to develop a voltage $\bar{V}$ when the cavity is tuned. The tuning angle $\varphi_{Z}$ is the argument of the complex impedance $Z_{\text {car }}$. The loading angle $\varphi_{\mathrm{L}}$ is the equivalent impedance angle as observed by the $\mathrm{RF}$ amplifier (phase angle between generator current $\overline{\mathrm{I}}_{\mathrm{G}}$ and voltage $\overline{\mathrm{V}}$ ).

For practical cases $\Delta f / f_{R}<1$. Then: $\tan \varphi_{Z} \sim 2 Q \Delta f / f_{R}$ where $Q$ is the quality factor of the resonator and $\Delta f=f_{R}-f_{C}$ is the detuning with respect to the $R F$ frequency $f_{C}$,

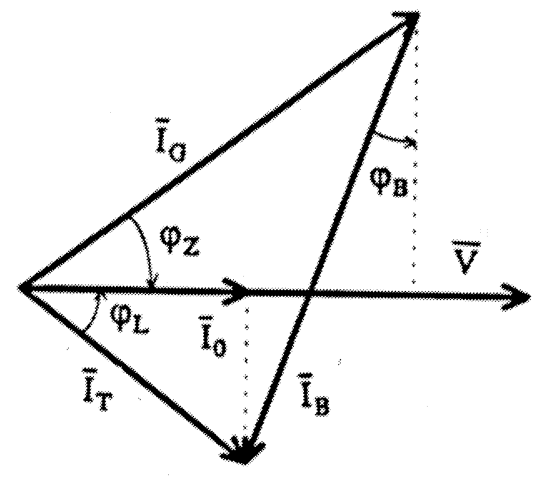

$I_{B}$ : peak beam current at $f_{C}, I_{B}=2 I_{D C}$ for short bunches, otherwise $I_{B}<2 I_{D C}, I_{D C}$ being the DC beam current

$\varphi_{B}$ : stable phase angle between RF components of beam current and cavity voltage $\varphi_{S}$ : stable phase angle for very short bunches. $\quad\left[I_{B} \sin \varphi_{B} / 2=I_{D C} \sin \varphi_{S}\right\}$

$\mathrm{Y}$ : by definition the relative beam loading $\mathrm{Y}$ is given by: $\mathrm{Y}=\mathrm{I}_{\mathrm{B}} / \mathrm{I}_{0}$

Figure 2: Steady state vector diagram below transition energy : $\quad \mathbb{\mathrm { I }}_{\mathrm{B}}$ downwards and with acceleration $\left(\varphi_{\mathrm{B}}>0\right): \quad \overline{\mathrm{I}}_{\mathrm{B}}$ leftwards 


\section{CONTINUOUS BEAM LOADING}

\subsection{ROBINSON INSTABILITY}

The cavity tune $\left(\mathrm{Z}_{\mathrm{cav}}\right)$ and the $\mathrm{RF}$ drive $\left(\mathrm{I}_{\mathrm{G}}\right)$ are assumed constant. Because of the vector addition of currents through the resonator (figure 2 ) any modulation of $\bar{I}_{B}$ induces changes in $\overline{\mathrm{I}}_{\mathrm{T}}$ and $\overline{\mathrm{V}}$, which in turn modulate the phase $\varphi_{\mathrm{B}}$ of $\overline{\mathrm{I}}_{\mathrm{B}}$. The stability limits of this closed loop system are of two different types:

- an impedance limitation. It is intensity independent and corresponds to in-phase dipolar oscillations of all bunches (mode $m=1 n=0$ ) [1].

- an RF power limitation. It is attained when the RF focusing is annihilated by the action of the beam current.

K.W. Robinson [5] derived rigorously the analytical criteria, and another derivation of the impedance limitation appears in this book [6]. This last limit can also be obtained directly from Sacherer's theory [1] applied to mode $m=1, n=0$. The analysis given below puts the emphasis on the illustration of the physical processes using deliberately some simplifications. The rigorous demonstration can be found in the above references.

\subsubsection{Impedance limitation}

Let us assume the case of a beam energy below transition, and a cavity with a moderate $Q$, such that its filling time $\tau$ is small compared to the period $T_{S}$ of synchrotron oscillation of the beam :

$$
\tau=\mathrm{Q} /\left(\pi \mathrm{f}_{\mathrm{R}}\right) \ll \mathrm{T}_{\mathrm{S}}=1 / \mathrm{f}_{\mathrm{S}}
$$

When a point charge makes a synchrotron oscillation around the synchronous particle, its frequency oscillates around the RF carrier frequency $\mathrm{f}_{\mathrm{C}}$ driving the cavity, At each moment it loses energy proportionally to the real part of the cavity impedance (figure 3). 
Qualitative approach

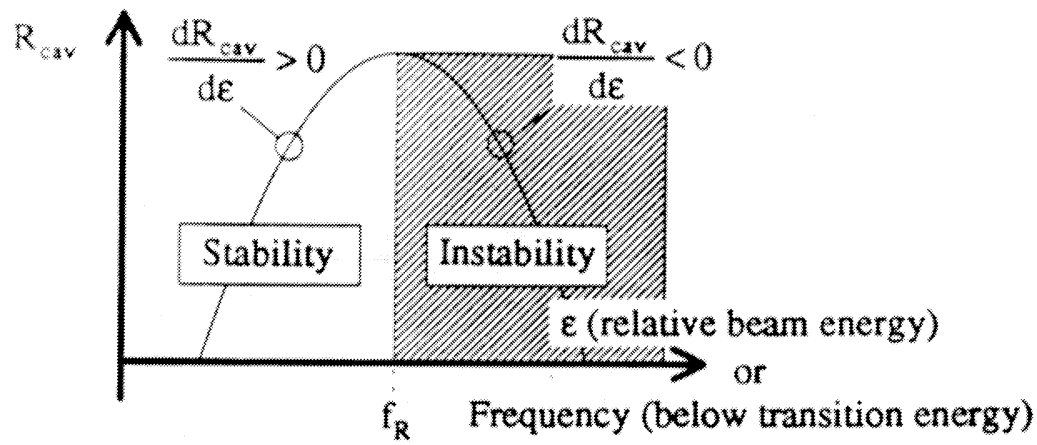

Figure $3:$ Impedance limitation below transition energy

- If the resonator is uned below the RF frequency $\left(f_{R}<f_{C}\right)$, the resistive part of the impedance decreases when frequency or energy increases (below transition a higher frequency corresponds to a higher energy). When the beam is at a higher energy than the synchronous particle, it dissipates less energy in the cavity. Conversely, when the beam is at a lower energy than the synchronous particle it loses more energy. The synchrotron oscillation amplitude is growing (anti-damping): there is instability.

- If the resonator is tuned above the RF frequency $\left(f_{R}>f_{C}\right)$ the reverse is true. The synchrotron oscillation amplitude decreases because of energy dissipation in the cavity. The system is stable.

The criterion is then : $\varphi_{Z}>0$ below transition energy, and it can be demonstrated to be the reverse above the transition energy.

\section{Quantitative approach}

Let us consider the synchrotron oscillation of a very short bunch of $\mathrm{N}$ particles in the longitudinal phase plane with the coordinate system $(\varphi, \varepsilon)$.

$\varphi$ is the phase of the RF component of the beam current with respect to the RF voltage, $\varepsilon=\Delta \mathrm{E} / \mathrm{E}$, 
$E$ is the synchronous particle energy, and $\Delta E$ the energy difference between beam and synchronous particle.

The voltage $V$ in the cavity changes the relative beam energy per charge with respect to the synchronous particle at a rate given by:

$$
\tilde{\varepsilon}_{1}=\frac{I_{D C} V\left(\sin \varphi-\sin \varphi_{S}\right)}{N E}
$$

We shall not consider the effect of the reactive part of the cavity impedance, since it does not contribute to the damping or anti-damping of the oscillation. Dissipation in the real part $R_{c a v}$ of the impedance of the cavity leads to a rate of loss of relative energy :

$$
\dot{\varepsilon}_{2}=-\frac{\mathrm{I}_{\mathrm{B}}^{2}}{2 \mathrm{NE}}\left(\frac{\mathrm{dR}}{\mathrm{d} \varepsilon}\right) \varepsilon \approx-\frac{2 \mathrm{I}_{\mathrm{DC}}^{2}}{\mathrm{NE}}\left(\frac{\mathrm{dR}_{\mathrm{cav}}}{\mathrm{d} \varepsilon}\right) \varepsilon .
$$

The total rate of change of $\varepsilon$ per charge is then :

$$
\dot{\varepsilon}=\frac{I_{D C} V\left(\sin \varphi-\sin \varphi_{S}\right)}{N E}-\frac{2 I_{D C}^{2}}{N E}\left(\frac{d R_{C W V}}{d \varepsilon}\right) \varepsilon
$$

In a synchrotron at a given B field, a relative frequency change $\Delta \omega / \omega$ is linked to a relative momentum difference $\Delta p / p$ by :

$$
\frac{\Delta \omega}{\omega}=-\eta \frac{\Delta p}{p}=-\frac{\eta}{\beta^{2}} \varepsilon \quad \text { using the relation: } \quad \frac{\Delta p}{p}=\frac{1}{\beta^{2}} \frac{\Delta E}{E}=\frac{1}{\beta^{2}} \varepsilon
$$

where $\eta=\frac{1}{\gamma_{\mathrm{T}}^{2}}-\frac{1}{\gamma^{2}}, \gamma=\frac{\mathrm{E}}{\mathrm{E}_{0}}$ and $\beta$ are the usual relativistic parameters, $\gamma_{\mathrm{T}}$ being the value of $\gamma$ at transition.:

Consequently: $\quad \dot{\varphi}=-\Delta \omega=\frac{\omega_{c} \eta}{\beta^{2}} \varepsilon$,

Differentiating (1) with respect to time, and using (2), the following second-order equation is derived: 


$$
\ddot{\varepsilon}+\frac{2 I_{D C}^{2}}{N E}\left(\frac{d R_{a \alpha}}{d \varepsilon}\right) \dot{\varepsilon}-\frac{I_{D C} V \cos \varphi_{S}}{N E} \frac{\omega_{C} \eta}{\beta^{2}} \varepsilon=0
$$

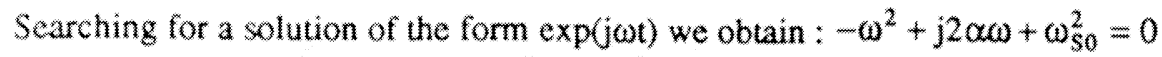

$$
\begin{aligned}
\text { using } \quad \alpha=\frac{I_{D C}^{2}}{N E}\left(\frac{d R_{\mathrm{CN}}}{d \varepsilon}\right) \\
\omega_{S 0}^{2}=-\frac{I_{D C} V \cos \varphi_{S}}{N E} \frac{\omega_{C} \eta}{\beta^{2}} .
\end{aligned}
$$

Roots of (4) are of the form : $\omega_{i}=j \alpha \pm \sqrt{\omega_{\mathrm{S} 0}^{2}-\alpha^{2}}$.

Solutions of (3) are linear combinations of $\mathrm{e}^{-\alpha t} \mathrm{e}^{+\left(\sqrt{\omega_{30}^{2}-\alpha^{2}}\right)}$ and $\mathrm{e}^{-\alpha \mathrm{t}} \mathrm{e}^{-\left(\sqrt{\omega_{30}^{2}-\alpha^{2}}\right) t}$

$\alpha$ is the damping rate of the synchrotron oscillation. Since $\alpha$ is of the sign of $\left(\frac{\mathrm{dR}_{\mathrm{cav}}}{\mathrm{d} \varepsilon}\right) \quad($ see $(5))$, stability can only be preserved for $\left(\frac{\mathrm{d} \mathrm{R}_{\mathrm{avv}}}{\mathrm{d} \varepsilon}\right)>0$.

For the system to be stable against the Robinson instability, the resistive part of the cavity impedance must be a growing function of energy. Below transition for instance, this means that the cavity tune must be higher than the RF frequency, as predicted with the preceding qualitative analysis.

Remark : $\alpha$ can be derived from the rigorous expression $[6]: \alpha=\frac{\omega_{S 0} I_{D C}\left(Z_{R}^{+}-Z_{R}^{-}\right)}{2 V \cos \varphi_{S}}$ where $\mathrm{Z}_{\mathrm{R}}^{+}, \mathrm{Z}_{\mathrm{R}}$ are the real part of the impedance at $\omega_{\mathrm{C}}+\omega_{\mathrm{SO}}$ and $\omega_{\mathrm{C}}-\omega_{\mathrm{so}}$ respectively.

\subsubsection{RF power limitation}

The total RF power delivered to the bunch(es) sitting at the phase $\varphi_{\mathrm{B}}$ is :

$$
P_{B}=\frac{V I_{B} \sin \varphi_{B}}{2}=\frac{R I_{B}^{2} \sin \varphi_{B}}{2 Y}
$$


using $\quad \mathrm{V}=\mathrm{RI}_{0}$ and $\mathrm{Y}=\frac{\mathrm{I}_{\mathrm{B}}}{\mathrm{I}_{0}}$.

Focusing of the bunch(es) towards the stable phase disappears when :

$$
\begin{gathered}
\frac{\mathrm{d} P_{\mathrm{B}}}{\mathrm{d} \varphi_{\mathrm{B}}}\left(@ \mathrm{I}_{\mathrm{G}}, \varphi_{\mathrm{Z}}, \mathrm{I}_{\mathrm{B}}=\text { const. }\right)=0 \\
\frac{\mathrm{d} P_{\mathrm{B}}}{\mathrm{d} \varphi_{\mathrm{B}}}=\frac{R \mathrm{I}_{\mathrm{B}}^{2}}{2}\left(\frac{\cos \varphi_{\mathrm{B}}}{\mathrm{Y}} \mathrm{d} \varphi_{\mathrm{B}}-\frac{\sin \varphi_{\mathrm{B}}}{\mathrm{Y}^{2}} \mathrm{dY}\right) .
\end{gathered}
$$

From geometrical considerations in figure 2 , we can write :

$$
\mathrm{I}_{\mathrm{G}}^{2}=\mathrm{I}_{\mathrm{T}}^{2}+\mathrm{I}_{\mathrm{B}}^{2}-2 \mathrm{I}_{\mathrm{T}} \mathrm{I}_{\mathrm{B}} \sin \left(\varphi_{Z}-\varphi_{\mathrm{B}}\right)
$$

which we transform into :

$$
\left(\frac{\mathrm{I}_{\mathrm{O}}}{\mathrm{I}_{\mathrm{B}}}\right)^{2}=1+\frac{1}{\mathrm{Y}^{2} \cos ^{2} \varphi_{Z}}-\frac{2 \sin \left(\varphi_{Z}-\varphi_{\mathrm{B}}\right)}{\mathrm{Y} \cos \varphi_{Z}} .
$$

Differentiating (9) with the constraint $\mathrm{I}_{0}, \varphi_{2}, \mathrm{I}_{\mathrm{B}}=$ constant leads to the relation :

$$
\mathrm{d} Y\left[Y \cos \varphi_{Z} \sin \left(\varphi_{Z}-\varphi_{\mathrm{B}}\right)-1\right]=\mathrm{d} \varphi_{\mathrm{B}}\left[-\mathrm{Y}^{2} \cos \varphi_{\mathrm{Z}} \cos \left(\varphi_{\mathrm{Z}}-\varphi_{\mathrm{B}}\right)\right]
$$

Bringing (10) into (8) and after some algebra we finally get :

$$
Y \sin 2 \varphi_{Z}=2 \cos \varphi_{B}
$$

which is precisely the second stability criterion from Robinson.

This second limit of stability is then clearly due to the limitation in the available RF power. When the threshold (11) is attained the instability grows exponentially, without oscillation, contrarily to the one due to the impedance limitation (section 3.1.1). 


\subsubsection{Complete Robinson criteria}

Below transition energy the full Robinson criteria [5] are :

$$
0<Y \sin 2 \varphi_{Z}<2 \cos \varphi_{B} .
$$

In the coordinate system $\left(\varphi_{Z}, Y\right)$ they take the form illustrated in figure 4 , for $\varphi_{\mathrm{B}}=0$.

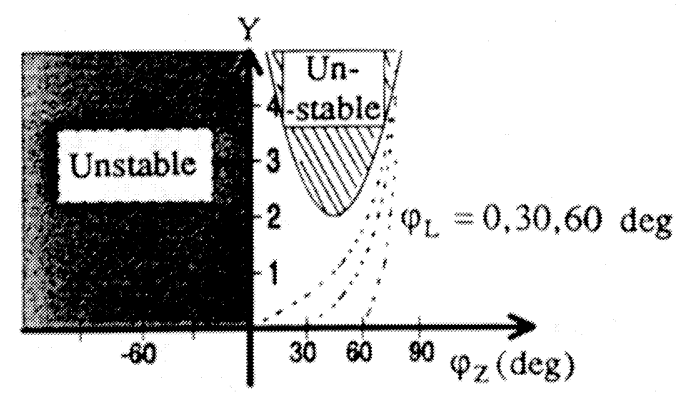

Figure 4 : Robinson stability limits for $\varphi_{\mathrm{B}}=0$

According to the previous analysis the $Y$ axis is the limit for an anti-damped oscillation around the stable particle ("Impedance limitation"), while the parabola in the first quadrant is the limit for an aperiodic type of movement away from the stable phase ("RF power limitation").

When a slow tuning system is active ("slow" meaning : much slower than the period of synchrotron oscillation) which controls the reactive power required from the amplifier $\left(\varphi_{\mathrm{L}}=\right.$ constant $)$, then :

$$
\tan \varphi_{\mathrm{Z}}=\mathrm{Y}\left(\cos \varphi_{\mathrm{B}}+\sin \varphi_{\mathrm{B}} \tan \varphi_{\mathrm{L}}\right)+\tan \varphi_{\mathrm{L}}
$$

This relation is represented by the dotted lines in figure 4 . It is clear that keeping $\varphi_{L}$ slightly positive is sufficient to guarantee stability. 
Remark: For a beam energy above transition, the sign of the inequalities in (12) is reversed. In the graphics of figure 4 , the picture is symmetrical to the one below transition with respect to the $Y$ axis.

\subsection{MULTI-LOOP INSTABILITY}

\subsubsection{Modelling}

Robinson criteria rarely apply to real RF systems because of the presence of various servo-loops for cavity tuning, gap voltage regulation, beam phase oscillation damping, etc... Neither $\bar{I}_{\mathrm{a}}$ nor $\varphi_{\mathrm{z}}$ are independent variables any more. Figure 5 shows these loops in the case of a typical RF system for a proton synchrotron.

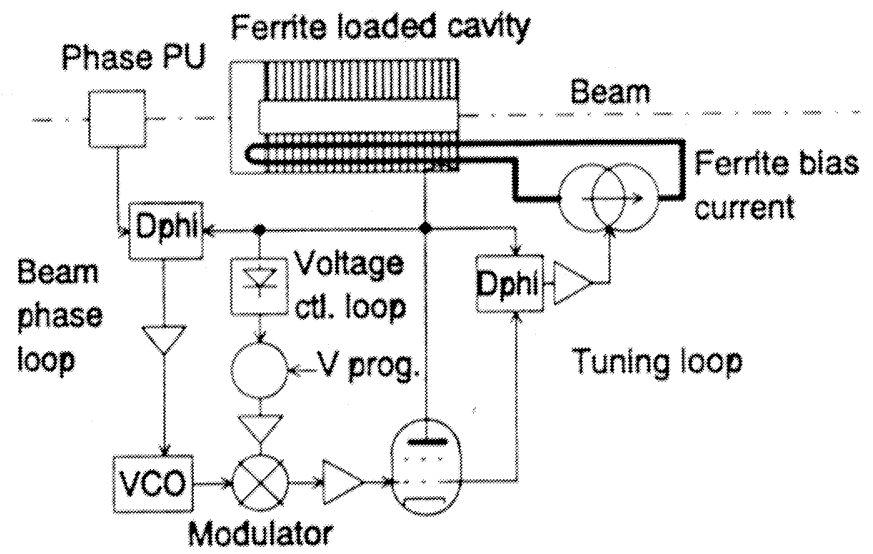

Figure 5 : Typical RF system for a proton synchrotron

\section{Small beam intensity}

The loops are coupled if the cavity is not tuned at the RF frequency because of Amplitude Modulation (AM) to Phase Modulation (PM) and PM to AM conversion in the detuned resonator. This is minimized by keeping the best possible tune and by choosing largely different cut-off frequencies. In such conditions each loop can be optimized for 
stability. In particular the beam phase loop damps the coherent oscillations of the bunch(es) (suppression of the "impedance limitation" leading to the instability in quadrant 2 of figure 4).

\section{Large beam intensity}

Because of vector addition of $\overrightarrow{\mathrm{I}}_{\mathrm{B}}$ with $\overline{\mathrm{I}}_{\mathrm{Q}}$ to form $\mathrm{I}_{\mathrm{T}}$ in the cavity, coupling is unavoidable between the loops. Stability analysis has to enclose all the loops and their coupling [4].

Small modulations of phase $\left(\mathrm{p}_{\mathrm{x}}\right)$ and relative amplitude $\left(\mathrm{a}_{\mathrm{x}}\right)$ of a variable $\mathrm{x}$ are defined as :

$$
x(t)=\operatorname{Re}\left[x\left(1+a_{x}(t)\right) e^{j\left(\omega_{c^{t}}+p_{x}(t)\right)}\right] \quad \text { with } a_{x}<<1 \text { and } p_{x}<<1 .
$$

For such modulations we can derive the flow graph in figure 6 (from [4]), where the following transfer functions (Laplace transforms) are used :

$C_{p}, C_{*}, C_{t}:$ Transmission through the low frequency electronics of the phase, amplitude and tuning loops

$\mathrm{G}_{p p}^{\mathrm{G}}\left(\mathrm{G}_{\mathrm{kn}}^{\mathrm{C}}\right)$ : Transmission of phase and amplitude modulation of $\overrightarrow{\mathrm{I}}_{\mathrm{G}}$ into phase (amplitude) modulation of $\bar{\nabla}$

$\mathrm{G}_{\mathrm{pa}}^{\mathrm{G}}\left(\mathrm{G}_{\mathrm{xp}}^{\mathrm{G}}\right)$ : Coupling of phase (amplitude) modulation of $\overline{\mathrm{I}}_{\mathrm{Q}}$ into amplitude (phase) modulation of $\overline{\mathrm{V}}$

$\mathrm{G}_{\mathrm{pp}}^{\mathrm{B}}\left(\mathrm{G}_{\mathrm{pa}}^{\mathrm{B}}\right)$ : Transmission of phase modulation of $\overline{\mathrm{I}}_{\mathrm{B}}$ into phase (amplitude) modulation of $\bar{V}$

$\mathrm{G}_{x p}\left(\mathrm{G}_{\mathrm{xa}}\right)$ : Transmission of tuning parameter modulation into phase (amplitude) modulation of $\bar{V}$

B : Transfer function of the phase of $\bar{\nabla}$ with respect to the stable phase through the beam and into the phase of $T_{\mathrm{B}} \quad B=\frac{\omega_{\mathrm{S}}^{2}}{\mathrm{~s}^{2}+\omega_{\mathrm{S}}^{2}}$.

The cavity impedance is : $Z(s)=\frac{2 \sigma R s}{s^{2}+2 \sigma s+\omega_{R}^{2}}$ 
where $\sigma=\frac{\omega_{R}}{2 Q}$ is the damping rate and $\omega_{R}$ the cavity resonant frequency (in rad/s).

$\omega_{R}, \omega_{C}, \sigma, \varphi_{Z}$ are linked by: $\omega_{R}-\omega_{C}=\sigma \tan \varphi_{Z}$

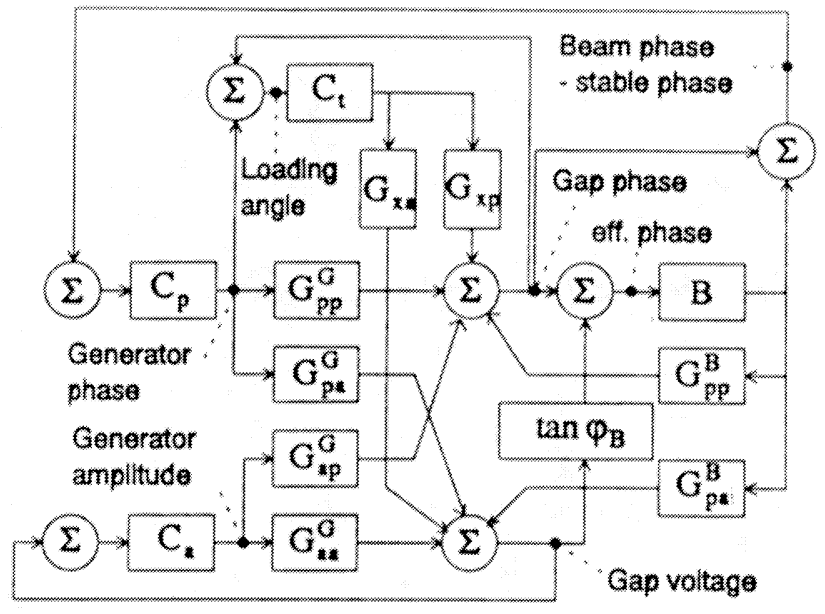

Figure 6: Flow graph for a complete RF system ( from F. Pedersen [4])

Transmission of small modulations of $\bar{I}_{\mathrm{T}}$ through the cavity impedance into modulations of $\overline{\mathrm{V}}$ are linear and given by:

$$
\begin{aligned}
& G_{p p}=G_{u}=\frac{1}{2}\left\{\frac{z\left(s+j \omega_{c}\right)}{Z\left(j \omega_{C}\right)}+\frac{Z\left(s-j \omega_{c}\right)}{Z\left(-j \omega_{C}\right)}\right\} \\
& G_{p k}=-G_{s p}=\frac{1}{2}\left\{\frac{z\left(s+j \omega_{C}\right)}{Z\left(j \omega_{C}\right)}-\frac{z\left(s-j \omega_{C}\right)}{Z\left(-j \omega_{C}\right)}\right\} .
\end{aligned}
$$

Using (15) and (17) we get :

$$
\begin{aligned}
& G_{p p}=G_{a k}=\frac{\sigma^{2}\left(1+\tan ^{2} \varphi_{z}\right)+\sigma s}{s^{2}+2 \sigma s+\sigma^{2}\left(1+\tan ^{2} \varphi_{Z}\right)} \\
& G_{p s}=-G_{x p}=\frac{\sigma \tan \varphi_{Z} s}{s^{2}+2 \sigma s+\sigma^{2}\left(1+\tan ^{2} \varphi_{Z}\right)} .
\end{aligned}
$$


$\mathrm{I}_{\mathrm{r}}$ being the vector sum of $\mathrm{I}_{\sigma}$ and $\mathrm{I}_{\mathrm{B}}$, we need to project properly their modulations on $\mathrm{I}_{\mathrm{T}}$ to get finally:

$$
\begin{aligned}
& G_{p p}^{G}=G_{A}^{G}=\frac{\sigma^{2}\left(1+\tan ^{2} \varphi_{Z}+Y\left(\sin \varphi_{B}-\tan \varphi_{Z} \cos \varphi_{B}\right)\right)+\sigma\left(1+Y \sin \varphi_{B}\right) s}{s^{2}+2 \sigma s+\sigma^{2}\left(1+\tan ^{2} \varphi_{Z}\right)} \\
& G_{p *}^{G}=-G_{\mathrm{ap}}^{\mathrm{O}}=\frac{-\sigma^{2} Y\left(\cos \varphi_{B}+\tan \varphi_{Z} \sin \varphi_{B}\right)+\sigma\left(\tan \varphi_{Z}-Y \cos \varphi_{B}\right) s}{s^{2}+2 \sigma s+\sigma^{2}\left(1+\tan ^{2} \varphi_{Z}\right)} \\
& G_{p p}^{B}=\frac{Y\left(\sigma^{2}\left(\tan \varphi_{Z} \cos \varphi_{B}-\sin \varphi_{B}\right)-\sigma \sin \varphi_{B} s\right)}{s^{2}+2 \sigma s+\sigma^{2}\left(1+\tan ^{2} \varphi_{Z}\right)} \\
& G_{p *}^{B}=-G_{a p}^{B}=\frac{Y\left(\sigma^{2}\left(\tan \varphi_{Z} \sin \varphi_{B}+\cos \varphi_{B}\right)+\sigma \cos \varphi_{B} s\right)}{s^{2}+2 \sigma s+\sigma^{2}\left(1+\tan ^{2} \varphi_{Z}\right)} .
\end{aligned}
$$

Letting the tuning control parameter $x$ be : $x=\frac{\Delta \omega_{\mathrm{R}}}{\sigma}$, we also get :

$$
\begin{aligned}
& G_{x p}=\frac{\sigma^{2}+\sigma s}{s^{2}+2 \sigma s+\sigma^{2}\left(1+\tan ^{2} \varphi_{z}\right)} \\
& G_{x a}=\frac{-\sigma^{2} \tan \varphi_{z}}{s^{2}+2 \sigma s+\sigma^{2}\left(1+\tan ^{2} \varphi_{z}\right)} .
\end{aligned}
$$

\subsubsection{Analysis}

Even with simple integrators in the electronics of the loops $\left(\mathrm{C}_{p}=\frac{\omega_{p}}{s}\right.$, etc... the characteristic equation of the system is already of degree 8 in s. The analytical treatment of the full system is very complicated, and the degrees of freedom are many. However numerical determination of the limits of stability is easy with a computer once the various parameters are known. 
With the simplifying assumptions $\varphi_{n}=0, \varphi_{k}=0, \sigma=0$ and neglecting transmission through the beam $(B(s)=0)$, the following criterion is obtained $[4]$ :

$$
Y<\sqrt{2+\frac{\omega_{k}}{\omega_{T}}+\frac{\omega_{\mathrm{T}}}{\omega_{*}}+\frac{\omega_{\mathrm{p}}}{\omega_{\mathrm{T}}}+\frac{\omega_{\mathrm{T}}}{\omega_{\mathrm{p}}}+\frac{\omega_{\mathrm{k}}}{\omega_{\mathrm{p}}}+\frac{\omega_{\mathrm{p}}}{\omega_{n}}}
$$

The stability limits in the case of a "typical" RF system for a proton synchrotron at $\varphi_{\mathrm{g}}=0$ and no tuning loop is shown in figure 7 for comparison with the no-loop situation corresponding to the Robinson criteria (figure 4).

$\frac{\omega_{s}}{2 \pi}=1.8 \mathrm{kHz} \quad \frac{3}{2 \pi}=50 \mathrm{kHz} \quad(1 / 2$ cavity $3 \mathrm{db}$ bandwidth $)$

$\frac{\omega_{g}}{2 \pi}=18 \mathrm{kHz} \quad \frac{\omega_{k}}{2 \pi}=6 \mathrm{kHz}$

$\frac{\omega_{\mathrm{T}}}{2 \pi}=0 \mathrm{kHz}$ (no tuning loop)

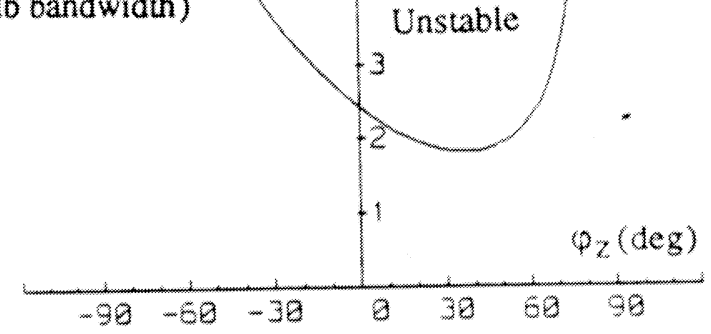

Figure 7 : Stability limits for a "typical" RF system with $\varphi_{\mathrm{B}}=0$

\section{Case of a very fast tuning loop}

It is worthwhile to note that in the case of a very fast tuning loop maintaining $\varphi_{\mathrm{L}}=0$, and with no other loop active, the instability limit is independent of $\varphi_{Z}$ and is simply given by:

$$
\mathrm{Y} \sin \varphi_{\mathrm{B}}<1
$$

which corresponds to an optimum transfer of power from the generator to the beam. It is an RF power limitation which can be derived with the procedure applied in section 3.1.2. 


\section{TRANSIENT BEAM LOADING}

\subsection{BASIC RELATIONS}

In the analysis of section 3 , low frequency (a few times $\mathrm{f}_{\mathrm{S}}$ ) modulations of small amplitude around the steady state have been considered. Consequently only rigid dipolar coherent oscillations $(m=1, n=0)$ of the beam have been taken into account. The situation is different when the system is perturbed by fast transients in the beam current, with a repetition period which is larger than the cavity filling time $\tau=1 / \sigma$. The spectral components of the beam current cover a wide frequency range where the cavity impedance is non-negligible. This is the case at injection or ejection, and also when the beam has gaps which are large with respect to t.

Contrarily to the steady-state case where an ideal current generator is the best model for the beam effect, the transient analysis is often easier when calculating voltages [7]. The passage of a point charge across the cavity loads the capacitor $\mathrm{C}$, which is equivalent to a step-like perturbation of $\mathrm{V}$.

\section{"Fundamental theorem of beam loading" [7]}

When a point charge crosses an empty cavity, it leaves behind a voltage $V_{b 0}$. The point charge itself is decelerated by $V_{b 0} 2$.

An intuitive demonstration of this effect can be given as follows. Since the charge is point like, $\mathrm{LdI}_{\mathrm{B}} / \mathrm{dt}$ is infinite and no energy is left in the inductance $\mathrm{L}$. The voltage $\mathrm{V}_{\mathrm{b} 0}$ developed across the capacitance $\mathrm{C}$ is related to the beam charge $\mathrm{q}$ by :

$$
v_{b 0}=\frac{q}{C}
$$

The energy stored in $\mathrm{C}$ is : $\quad \Delta \mathrm{U}_{\mathrm{C}}=\frac{1}{2} \mathrm{CV}_{\mathrm{b} 0}^{2}$. 
Letting $V_{D E C}$ be the decelerating voltage experienced by the charge $q$, the energy lost by the beam is:

$$
\Delta \mathrm{U}_{0}=\mathrm{qV}_{\mathrm{beC}}=\mathrm{CV}_{\mathrm{b} 0} \mathrm{~V}_{\mathrm{DEC}}
$$

Since the energy lost by the charge $\Delta \mathrm{U}_{0}$ has been transferred to the capacitance $\mathrm{C}$ as stored energy $\Delta U_{C}$;

$$
\Delta \mathrm{U}_{0}=\Delta \mathrm{U}_{\mathrm{C}} \Rightarrow \mathrm{V}_{\mathrm{DEC}}=\frac{\mathrm{V}_{\mathrm{b} 0}}{2}
$$

\section{Transient response to a single beam passage}

When a point charge crosses the cavity gap at time $t=0 \mathrm{~s}$, the voltage $\mathrm{V}$ starts a damped oscillation at the cavity resonance frequency $\omega_{\mathrm{R}}$ (in rad/s), with a damping rate $\sigma=1 / \tau$ (in $s^{-1}$ ), the initial value being $\mathrm{V}_{\mathrm{b} 0}$ :

$$
\bar{V}=V_{b 0} e^{\left(-\sigma+j 0_{n}\right) t} .
$$

If a voltage $V_{g}$ at frequency $\omega_{C}$ is forced across the gap by the RF amplifier before the beam passage, the result is simply the vector sum of both voltages.

\subsection{NON-PERIODIC CURRENT TRANSIENTS}

Such transients are encountered at beam transfer (injection/ejection), or while crossing transition energy in proton accelerators for instance. These disturbances being rare (a few per machine cycle) they correspond to step-like perturbations, and the usual servo-loops can be sufficient to get a properly damped response. The loop parameters must be such that the full RF system is stable under the analysis of section 3.2. On top of that the RF amplifier must be powerful enough to cope with the situation until the tuning loop has settled to the new optimum. This matter is treated in section 6.

Let us assume a periodic beam traversal with a period $T_{b}$ (evenly spaced bunches of equal intensity), and a voltage $\bar{V}_{\mathrm{g}}$ at frequency $\omega_{\mathrm{C}}$ forced across the unperturbed cavity by the 
RF amplifier. Taking the phase of $\overline{\mathrm{V}}_{\mathrm{g}}$ as reference, the voltage seen by the beam after many traversals is given by $[7]$ :

$$
\left.\bar{V}=\bar{V}_{a}+V_{b 0} e^{-\left(\frac{\pi}{2} \cdot \varphi_{3}\right.}\right)\left(\frac{1}{2}+e^{\left(-\sigma+j \Delta(0) T_{b}\right.}+e^{2(-a+j \Delta \omega) T_{b}}+\ldots\right)
$$

where : $\quad \Delta \omega=\omega_{\mathrm{R}}-\omega_{\mathrm{C}}$.

This vector composition is shown in figure 8. Some algebraic transformations lead to :

$$
\left.\bar{V}=\bar{V}_{g}+\frac{V_{b 0}}{2} e^{-\left(\frac{*}{2}+\varphi_{s}\right.}\right)\left(\frac{1+e^{(-\sigma+j \omega \omega) \mathrm{r}_{\mathrm{b}}}}{1-\mathrm{e}^{(-\alpha+j \omega \omega) \mathrm{T}_{\mathrm{b}}}}\right)
$$

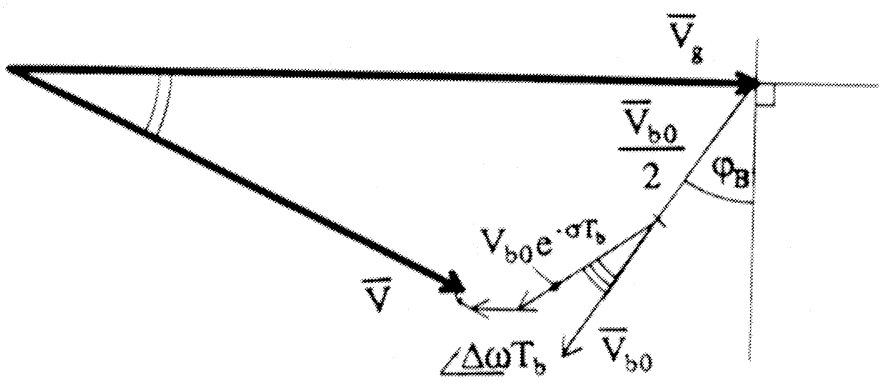

Figure 8 : Vector addition of voltages for repetitive bunch passages

After a transient period (a few times $\tau$ ) following the beginning of the bunch train, a stationary situation is attained, and all bunches experience the same voltage $\mathrm{V}$. Such a case is quasi-steady-state and can be handled as in section 3, although the gap voltage is clearly modulated at the bunch frequency $1 / \mathrm{T}_{\mathrm{b}}$.

\subsection{PERIODIC CURRENT TRANSIENTS}

Such a situation occurs when the spacing between bunches is unequal, or when the spacing is regular but not the intensity of the bunches. We shall illustrate this with the example of a batch of five adjacent bunches filling one half of the circumference $\left(h_{R F}=10\right)$. If $\tau<<\mathrm{T}_{\mathrm{b}}$ all transients have decayed between the bunches and the situation is comparable to the 
non-periodic transient case (section 4.2). If not, and if $\tau<\mathrm{T}_{\mathrm{REV}}$ the system is in a periodic transient case. Assuming $\tau<<T_{\text {REV }}$ then no voltage is left from the previous beam passage when the first bunch of the batch arrives. The first bunch sees $\vec{\nabla}_{b 1}$, the second $\vec{\nabla}_{b 2}$, etc.. according to (26):

$$
\begin{aligned}
& \bar{V}_{b 1}=\bar{V}_{s}+V_{b 0} e^{-\left(\frac{\pi}{2}+\phi_{a}\right)}\left(\frac{1}{2}\right) \\
& \bar{V}_{b 2}=\bar{V}_{b 1}+V_{b 0} e^{-\left(\frac{\pi}{2}+\omega_{1}\right)} e^{(-\sigma+j \alpha \omega) r_{b}} \\
& \bar{V}_{b 3}=\bar{V}_{b 2}+V_{b 0} e^{-\left(\frac{\pi}{2}+a_{2}\right)} e^{2(-\sigma+j)(\omega 0) r_{b}} \\
& \text { etc.... }
\end{aligned}
$$

Figure 9 shows the composition of vectors for the first two bunches. The successive bunches see voltages affected by phase and amplitude modulations.
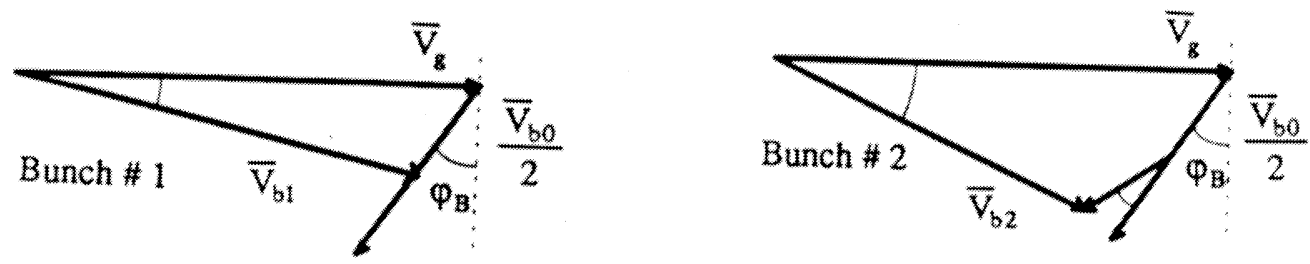

Figure 9 : Voltage seen by successive bunches

This result can also be derived from a frequency domain analysis, by complex addition of the voltages developed in the cavity impedance by the various spectral components of the beam current. Figure 10 shows the frequency domain situation alongside its time domain counterpart.

The important parameter is the cavity impedance at the various harmonics of the revolution frequency where large beam current components exist. The gap voltage $\mathrm{V}$ is modulated in amplitude and phase at a rate which is too fast and out of control for all the conventional loops envisaged in section 3.2. The amplifier works on a "pulsed" reactive load. 


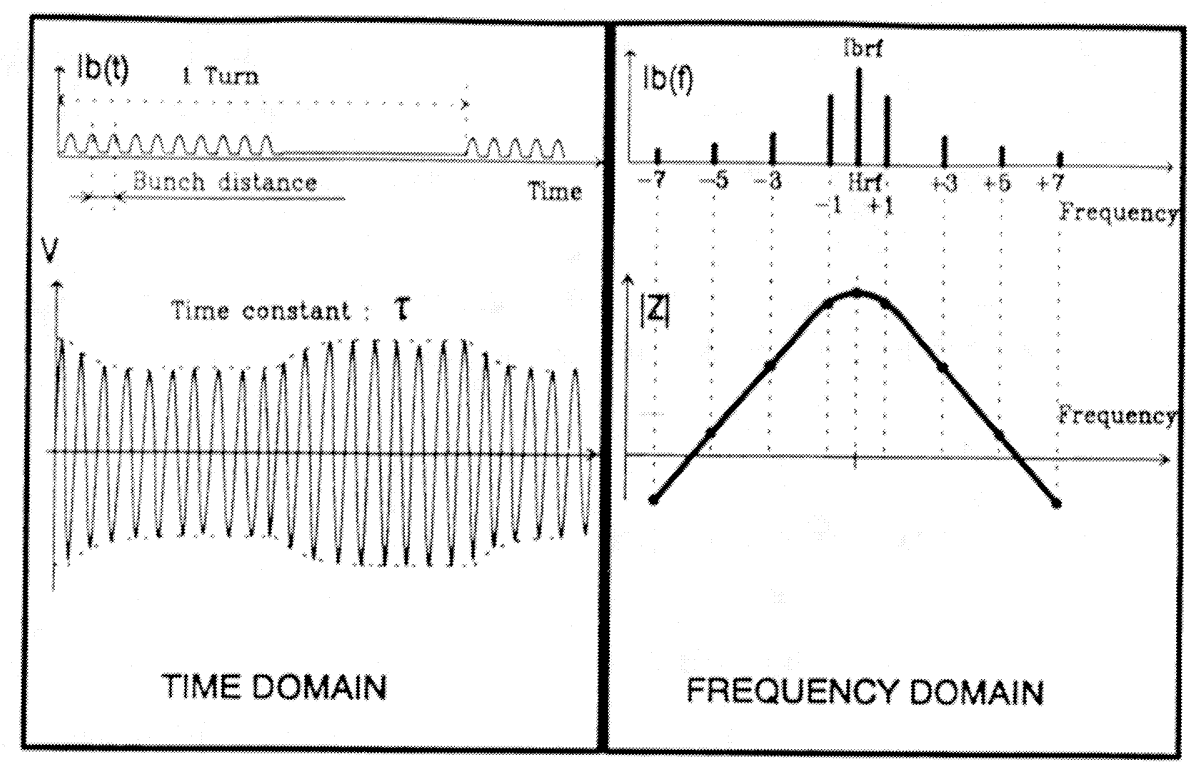

Figure $10:$ Time and frequency domain analysis

The successive bunches of the batch experience different longitudinal focusing voltages. The excitation of non-coherent beam oscillations is favoured, leading to longitudinal emittance blow-up after filamentation.

\section{CURES}

\subsection{PASSIVE DAMPING}

The brute force method to decrease the relative beam loading $Y=I_{B} / I_{0}$ is to increase $\mathrm{I}_{0^{*}}$. The immediate consequence is the requirement for more RF power from the amplifier, because of the higher dissipation in the resistance of the equivalent resonator. Various practical means can be applied for this purpose, such as : 
- using a low output impedance amplifier (triode, tetrode with a pulsed bias [8], amplifier overcoupled to the cavity when driving from a transmission line...)

- puting a dummy load in parallel with the cavity.

The performance obtained is easily estimated in stability diagrams as in figures 4 and 7 by scaling down the $Y$-axis values proportionally to the increase of $1_{0^{*}}$

\subsection{FEEDFORWARD $[9,10]$}

If the RF amplifier is driven to inject continuously a current $-\bar{I}_{\mathrm{B}}$ in the cavity, the equivalent impedance seen by the beam is zero. This technique is illustrated in figure 11 , where ${ } \bar{I}_{B}$ is added to the low level RF drive $\bar{I}_{G}$ to get the generator current $\bar{I}_{G}$.

$$
\left.\begin{array}{l}
\overrightarrow{\mathrm{I}}_{\mathrm{o}}=\overrightarrow{\mathrm{I}}_{\mathrm{O}}-\overrightarrow{\mathrm{I}}_{\mathrm{B}} \\
\overrightarrow{\mathrm{I}}_{\mathrm{T}}=\overrightarrow{\mathrm{I}}_{\mathrm{o}}+\overrightarrow{\mathrm{I}}_{\mathrm{B}}
\end{array}\right\} \Rightarrow \overrightarrow{\mathrm{I}}_{\mathrm{T}}=\overrightarrow{\mathrm{I}}_{\mathrm{G}}
$$
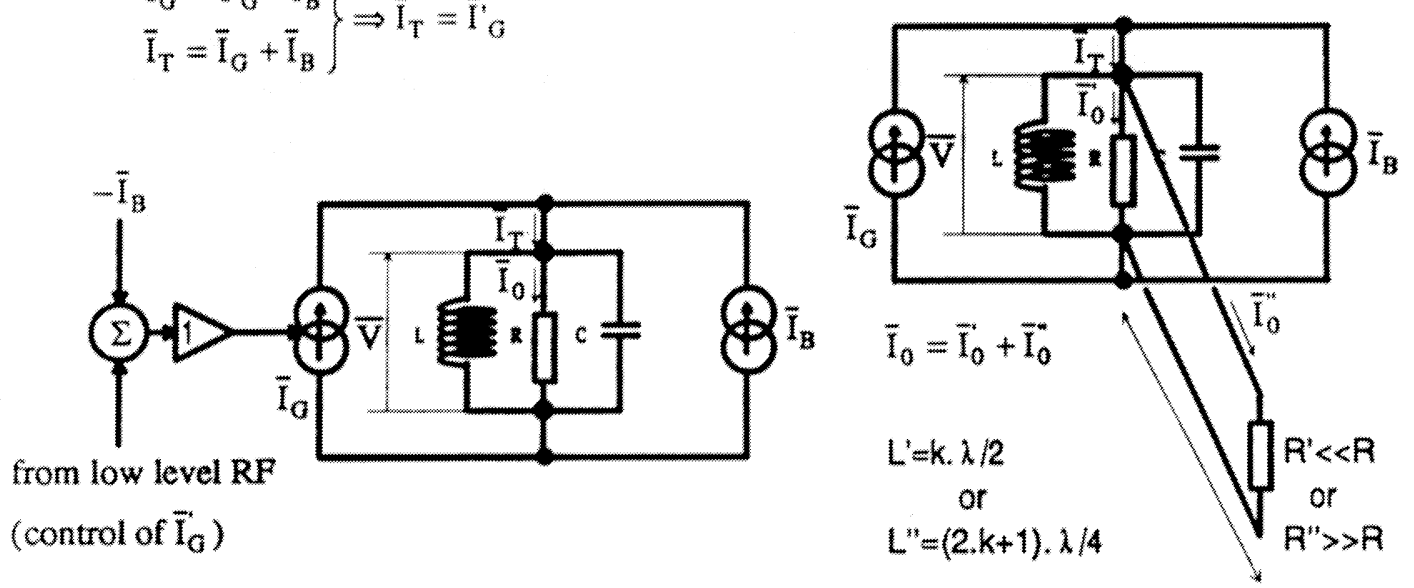

Figure 11 : Lay-out of a feedforward

Figure 12: Feedforward variant beam loading compensation

Another implementation of the same principle is possible, making use of the total reflection of the beam induced signal on the output of a strongly mismatched amplifier (figure 12). The electrical length of the transmission line is adjusted so that there is a voltage node in 
he standing wave pattern at the input port of the cavity $(2 k+1) \lambda / 4$ for an open circuit on the anplifer side).

The net result is that the low level RF system (Beam Control) controls directly $\vec{I}_{\mathrm{T}}$, instead of $T_{G}$ in the ordinary case. The cross-coupling between loops is considerably reduced and the global stability of the system is largely improved [9]. (The cross-coupling is not fully suppressed since modulations of $\mathrm{I}_{\mathrm{B}}$ still induce modulation of the loading angle $\varphi_{\mathrm{L}}$ regulated by the tuning loop.) The requirements for the high power hardware are unchanged.

\subsection{WIDE BAND FEEDBACK [11]}

Enclosing the high power amplifier and the cavity in a closed loop with an important loop gain (see figure 13) brings in the usual benefit of reducing the sensituvity of any controlled parameter versus an external perturbation. In particular the equivalent impedance seen by the beam $\left(Z_{\propto q}=d V / d_{B}\right)$ is changed according to:

$$
Z_{c q}=\frac{Z_{c a r}(j \omega)}{1+G_{o L}(j \omega)}
$$

where $\mathrm{G}_{\mathrm{OL}}(j \omega)$ is the loop gain. In a first approximation $\mathrm{Z}_{\mathrm{car}}$ is the only frequency sensitive clement in $\mathrm{G}_{\mathrm{OL}}(\mathrm{j} \omega)$. The loop gain is then maximum and real at the cavity resonance $\omega_{\mathrm{R}}$. where we get:

$$
\begin{gathered}
G_{O L}\left(j \omega_{R}\right)=A g R H \\
R_{e q}=\frac{R}{1+G_{O L}\left(j \omega_{R}\right)} \ll R .
\end{gathered}
$$

The transfer function from the low level input $v$ to the gap voltage $V$ at $\omega_{\mathrm{R}}$ is :

$$
\frac{V}{v}=\frac{A g R}{1+A g R H}=\frac{1}{H} \text {. }
$$

This demonstrates one very interesting feature of this technique : the low level system directly controls the gap voltage $V$, which is the most relevant physical parameter for the beam. 


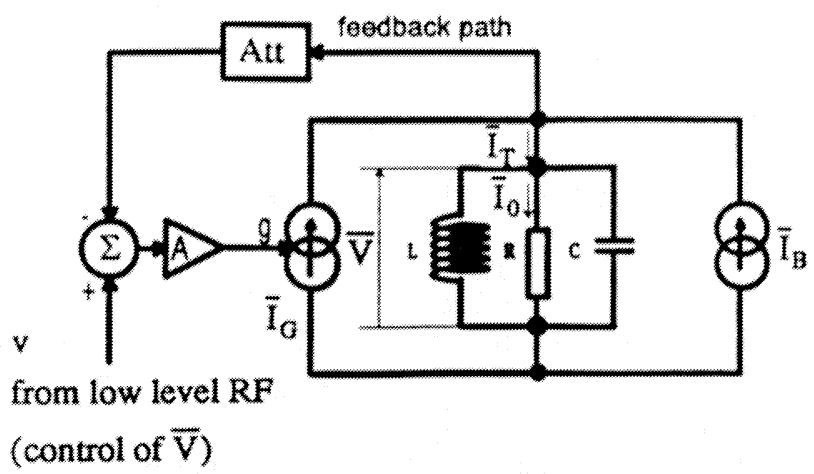

Figure 13: Lay-out of wide-band feedback

However the group delay $\tau$ in the loop must be tightly controlled. Let us assume a fixed frequency cavity, where the loop gain has to be larger than $0 \mathrm{~dB}$ over a bandwidth $\mathrm{B}$ (in $\mathrm{rad} / \mathrm{s})$ :

$$
\mathrm{B}>\omega_{3 \mathrm{~dB}}=\frac{\omega_{\mathrm{R}}}{\mathrm{Q}}=2 \sigma .
$$

The phase shift over this frequency range cannot exceed $3 \pi / 2 \mathrm{rad}$ if a phase margin of $\pi / 4 \mathrm{rad}$ is requested at $0 \mathrm{~dB}$ gain. Since the resonator itself contributes already for $\pi$ radians, then the phase shift due to $\tau$ must be smaller than 2 times $\pi / 4 \mathrm{rad}$. over $\mathrm{B} \mathrm{rad} / \mathrm{s}$ : $\quad B \tau<\frac{\pi}{2} \mathrm{rad}$.

From the approximate expression of the cavity impedance, far from resonance :

$$
\mathrm{Z}_{\mathrm{cuv}}\left(\mathrm{j}\left(\omega_{\mathrm{R}}+\Delta \omega\right)\right)=\frac{\mathrm{R}}{2 \mathrm{jQ} \frac{\Delta \omega}{\omega_{\mathrm{R}}}}
$$

we deduce that the maximum loop gain at resonance is :

$$
G_{O L \max }\left(j \omega_{R}\right)=\frac{\pi Q}{2 \omega_{R} \tau}
$$

and the corresponding minimum impedance at resonance is :

$$
R_{\text {eqmin }}=\frac{2 \omega_{R}}{\pi} \frac{R}{Q} \tau
$$

It is worthwhile to notice that this result only depends on R/Q (cavity geometry) and $\tau$. 
If the cavity is tunable over a frequency range

$$
B_{\text {Tuxe }}=\omega_{R \text { max }}-\omega_{\mathrm{Rmin}} \text {, }
$$

the requirement for the delay $t$ is even more stringent. With the same request for the phase

margin one needs:

$$
\left(\mathrm{B}_{\mathrm{Tune}}+\mathrm{B}\right) \tau<\frac{\pi}{2} \mathrm{rad}
$$

leading to a minimum impedance at resonance given by:

$$
R_{\text {eqmin }}=\frac{\omega_{R}}{2 \pi} \frac{R}{Q}\left(\frac{1}{\frac{1}{4 \tau}-B_{\text {Tune }}}\right)
$$

which is always less favourable than the fixed frequency optimum (35). A tunable low $Q$ resonator in the amplifier chain is a convenient means to ease the practical realization [12].

Implementing $R F$ feedback is equivalent to increasing $l_{0}$ by the factor $\left(1+\mathrm{G}_{\mathrm{OL}}\left(\mathrm{j} \omega_{\mathrm{R}}\right)\right)$, and consequently to reduce the relative beam loading $Y$ in the same proportion. The effect is the same than for passive damping (section 5.1), but no more power is required from the amplifier.

\section{Spurious effect of RF feedback}

The longitudinal coupled bunch instabilities threshold [1] is degraded for two main reasons:

- the real part of $Z_{e q}$, which is the determining factor for the instabilities, is increased outside the loop bandwidth, because of the coefficient $\frac{1}{\left(1+\mathrm{G}_{\mathrm{OL}}\left(j \omega_{R}\right)\right)}$ in the expression (30) of Zoq.

- if the phase shift in the amplifier chain is not ideally controlled to make $\mathrm{G}_{\mathrm{OL}}\left(\mathrm{j} \omega_{\mathrm{R}}\right)$ real , the maximum of $Z_{e q}$ is shifted in frequency. The real part of $Z_{\infty q}$ becomes asymmetrical with respect to the RF frequency, which combines with the first effect mentioned to help trigger beam instabilities. 
However a conventional multipath damping system [13] can still be used for compensation, if one takes properly into account the modification of the low level to gap voltage transfer function [14].

\subsection{LONG DELAY FEEDBACK $[15,16]$}

Moderate bandwidths of a few times the synchrotron frequency are of interest when considering the voltage induced on revolution harmonics different from the RF frequency. A feedback with a long delay can be considered, as long as the stability requirements are met. This is the case when the feedback path contains a comb filter with gain maxima at the revolution harmonics, and when the total electrical delay is made equal to one revolution period in the machine (Figure 14).

The long delay makes it possible to make the necessary hardware at low power level, where digital electronics can be used, and to install it outside the radiation area in a convenient equipment room.

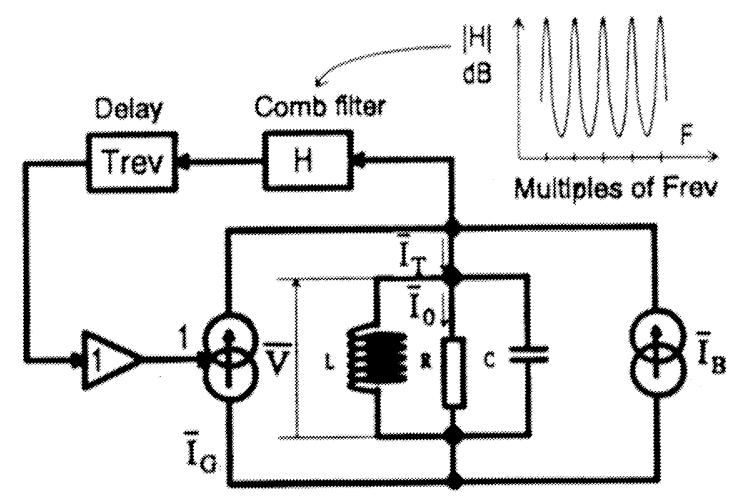

Figure 14 * Lay-out of "One turn delay" feedback

A simple recursive filter gives the following comb-like transfer function :

$$
H(j \omega)=\frac{\mathrm{G}_{0}}{1-\mathrm{Ke}^{-j \omega \mathrm{T}_{\operatorname{mav}}}}
$$


where $K$ and $G_{0}$ are constants : $0<K<1$, and $\Delta \omega=\omega-\omega_{R}$. Cascaded with the one turn delay $\left(e^{-j \Delta \omega T_{R E V}}\right)$ and the cavity impedance $Z_{c a v}(\omega \omega)$, the overall open loop transfer function is :

$$
G_{O L}(j \omega)=\frac{G_{0}}{e^{j \omega \omega T_{R E V}}-K} Z_{C \omega r}(j \omega)
$$

With $Z_{\text {car }}(j \omega)$ constant and real (case of a travelling wave cavity for instance [15]), it is represented in the complex plane by a circle centered on the real axis and crossing it at $\left[\frac{-G_{0} Z}{1+K}, 0\right]$ and $\left[\frac{G_{0} Z}{1-K}, 0\right]$ (figure 15 ).

Stability is preserved as long as $\frac{G_{0} Z}{1+K}<1$. The equivalent impedance for the beam is then :

$$
Z_{\propto q}(j \omega)=Z_{c a v}(j \omega)\left(\frac{e^{j \omega \omega T_{k R v}}-K}{e^{j \omega \omega T_{k e v}}-K+G_{0} Z_{c a v}(j \omega)}\right) .
$$

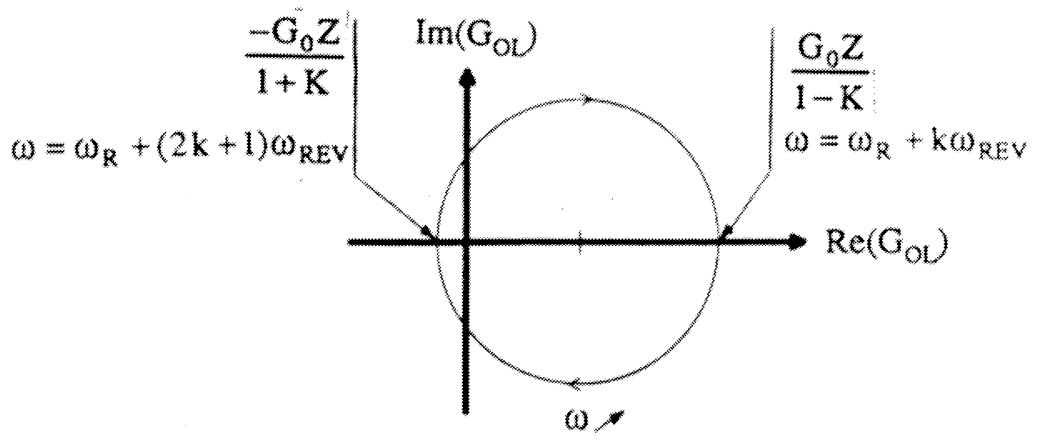

Figure 15: Open loop transfer function

Minima of $Z_{\propto q}$ occur at the revolution harmonics :

$$
\omega=\omega_{\mathrm{R}}+\mathrm{k} \omega_{\mathrm{REV}} \rightarrow \mathrm{Z}_{\mathrm{eq} \min }=\mathrm{Z}_{\mathrm{cav}}\left(\frac{1-\mathrm{K}}{1-\mathrm{K}+\mathrm{G}_{0} \mathrm{Z}_{\mathrm{cav}}}\right)=\frac{1-\mathrm{K}}{\mathrm{G}_{0}} \ll \mathrm{Z}_{\mathrm{cav}} .
$$

while impedance maxima are at : 


$$
\omega=\omega_{R}+(2 k+1) \omega_{R E V} \rightarrow Z_{\text {eqmax }}=Z_{\text {cav }}\left(\frac{1+K}{1+K-G_{0} Z_{\text {cav }}}\right)
$$

$$
\text { Selecting: } Z_{\text {eqmax }}=2 Z_{\text {s*v }} \Rightarrow \frac{G_{0} Z_{\text {cav }}}{1+K}=\frac{1}{2}
$$

the minimum impedance attained (43) is : $Z_{\text {cqmin }} \approx Z_{\text {cav }}(1-K)$ since $K=1$.

For smaller cavity bandwidth (non-constant $Z_{c a v}(j \omega)$ ) stability is still possible, and equivalent results are obtainable. A recent practical implementation of this principle has been made at the CERN PS, where the beam revolution frequency changes by $15 \%$ from injection to high energy, and the cavity is tunable from 4 to $9.5 \mathrm{MHz}$ (harmonic number from 10 to 20 ) [16].

The long delay feedback technique reduces the cavity impedance on many revolution harmonics, which is very efficient against periodic transient beam loading and longitudinal coupled-bunch instabilities (section 4.3).

The high power amplifier has to be able to provide the current requested at these frequencies, where its load is strongly mismatched (in fact the load is like a short circuit when the system works properly, since current is injected and no voltage is developed).

\subsection{COMPARISON OF CURES}

The characteristics of the various cures are gathered in Table 1. The wide band feedback technique is clearly the most interesting solution, as demonstrated by the number of developments in this direction over the recent years. A long delay feedback is a useful complement to reduce the impedance over a larger number of revolution harmonics than the wide band method alone can cover. 


\begin{tabular}{|l|l|l|l|l|}
\hline & $\begin{array}{l}\text { Passive } \\
\text { damping }\end{array}$ & Feedforward & $\begin{array}{l}\text { Wide band } \\
\text { feedback }\end{array}$ & $\begin{array}{l}\text { Long delay } \\
\text { feedback }\end{array}$ \\
\hline $\begin{array}{l}\text { References } \\
\text { continuous } \\
\text { beam loading }\end{array}$ & 8 & $9,10,20$ & $11,12,17,18,19$ & 15,16 \\
\hline $\begin{array}{l}\text { Effect on } \\
\text { transient beam } \\
\text { loading }\end{array}$ & Weak & $\begin{array}{l}\text { Delicate and } \\
\text { limited in } \\
\text { performance }\end{array}$ & $\begin{array}{l}\text { Positive but } \\
\text { function of loop } \\
\text { gain }\end{array}$ & $\begin{array}{l}\text { Non* } \\
\text { relevant }\end{array}$ \\
\hline $\begin{array}{l}\text { Spurious } \\
\text { effect on beam }\end{array}$ & None & $\begin{array}{l}\text { Marginally } \\
\text { positive }\end{array}$ & $\begin{array}{l}\text { Degrades instab. } \\
\text { threshold }\end{array}$ & $\begin{array}{l}\text { Improves instab. } \\
\text { threshold }\end{array}$ \\
\hline $\begin{array}{l}\text { Hardware } \\
\text { complexity }\end{array}$ & Small & Small to medium & Large & Medium \\
\hline $\begin{array}{l}\text { Run-time } \\
\text { adjustment }\end{array}$ & $\begin{array}{l}\text { Easy } \\
\text { (on bench) }\end{array}$ & $\begin{array}{l}\text { Lengthy } \\
\text { (with beam) }\end{array}$ & $\begin{array}{l}\text { Easy } \\
\text { (on bench) }\end{array}$ & $\begin{array}{l}\text { Easy } \\
\text { (on bench) }\end{array}$ \\
\hline $\begin{array}{l}\text { Stability } \\
\text { against drift }\end{array}$ & Good & $\begin{array}{l}\text { Bad } \\
\text { (open loop) }\end{array}$ & $\begin{array}{l}\text { Good } \\
\text { (closed loop) }\end{array}$ & $\begin{array}{l}\text { Good } \\
\text { (closed loop) }\end{array}$ \\
\hline $\begin{array}{l}\text { Oper. range } \\
\left(\text { Band } \mathrm{h}_{\mathrm{RP}}\right)\end{array}$ & Unlimited & $\begin{array}{l}\text { Lim. for good } \\
\text { performance }\end{array}$ & Unlimited & Unlimited \\
\hline Cost & Large & Small & Medium & Small \\
\hline $\begin{array}{l}\text { Oper. from } \\
\text { low level } \text { RF }\end{array}$ & Easy & Complex & Easy & Easy \\
\hline
\end{tabular}

Table 1 : Comparison of cures

Remark: Comments on the feedforward method assume that the beam signal is fed at the low power end of the amplification chain (long delay). Better ratings can be given when a small overall electrical delay is used[19].

\section{RF POWER REQUIREMENTS}

The two kinds of regimes investigated previously have to be analyzed to derive what is expected from the amplifier-cavity system and their associated equipment. The steady-state situation helps define the coupling factor and the CW RF power. Analysis of the transient 
cases gives indications on the peak capabilities needed (RF power, generator current, mismatch,...) and on the speed of the servo-loops (tuning and voltage). These aspects are treated in the following paragraphs for the two most frequently used amplifier-cavity set-ups.

\subsection{CURRENT GENERATOR NEAR TO THE CAVITY}

This is the case of medium power systems (up to $100 \mathrm{~kW}$ ), where the anode of a high power RF tube directly drives the cavity through a short connection. The vector diagram in figure 2 is used for the analysis.

\section{Steady-state operation}

When the tuning is not optimum $\left(\varphi_{L} \neq 0\right)$ the current $L_{G}$ is given by :

$$
\mathrm{I}_{\mathrm{G}}=\frac{\mathrm{I}_{0}+\mathrm{I}_{\mathrm{B}} \sin \varphi_{\mathrm{B}}}{\cos \varphi_{\mathrm{L}}}
$$

under the voltage $V$, which means the amplifier delivers a real power : $P_{\text {real }}=\frac{V I_{G} \cos \varphi_{L}}{2}$ and a reactive power: $\mathrm{P}_{\text {reactive }}=\frac{\mathrm{VI}_{\mathrm{C}} \sin \varphi_{\mathrm{L}}}{2}$.

With the optimum cavity tuning $\left(\varphi_{\mathrm{L}}=0\right)$ the current $\mathrm{L}_{\mathrm{G}}$ and the real power $P_{\text {real }}$ needed from the amplifier are minima. However, since : $I_{G}=I_{0}+l_{B} \sin \varphi_{B}$, the apparent load impedance is a function of $\mathrm{I}_{B}$ when $\varphi_{B} \neq 0$.

\section{Transient analysis}

Let us assume that the voltage and beam phase loops are fast enough to correct the gap voltage in amplitude and phase in a fraction of a synchrotron period. For a step-like perturbation $\overline{\Delta I}_{\mathrm{B}}$ in the beam current $\overline{\mathrm{I}}_{\mathrm{BO}}$ (case of section 4.2 ) the amplifier must "instantaneously" (faster than a synchrotron period) provide : $-\bar{\Delta}_{\mathrm{B}}+\bar{I}_{\mathrm{CO}}\left(\overline{\mathrm{I}}_{\mathrm{BO}}\right.$ and $\overline{\mathrm{I}}_{\mathrm{CO}}$ being the 
values before the perturbation). It lasts as long as the tuning loop has not settled again to $\varphi_{\mathrm{t}}=0$ : the faster the tuning loop, the shorter the requirement.

To cancel the effects of periodic transient beam loading (section 4.3 ) $l_{\mathrm{G}}$ has to be equal to the beam current at the various harmonics of the revolution frequency. The amplifier load at these frequencies is very low impedance when the system works properly (beam current properly compensated).

\subsection{AMPLIFIER AND ISOLATOR SET-UP [21]}

This is the case of very high power systems ( $-\mathrm{MW})$ used in large and very large synchrotrons, where RF is sent via a transmission line to the cavity, and tolerance to reflected power is limited. An isolator is inserted to guarantee a matched load to the amplifier (figure 16). RF power is then the only parameter to specify for the amplifier.

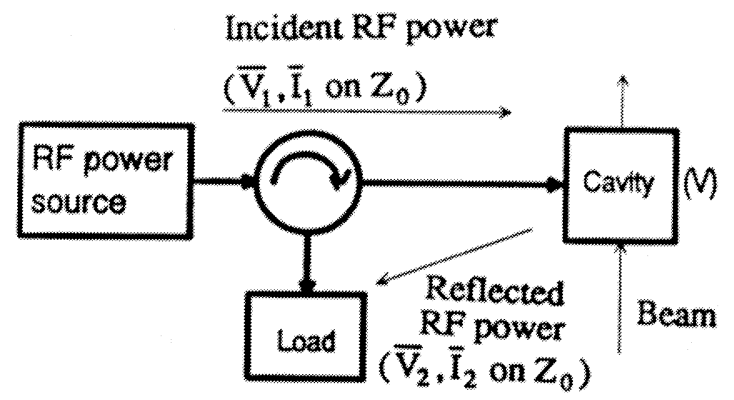

Figure 16: Amplifier with isolator set-up

To get the required voltage $\bar{V}$ in the cavity, the incident RF power (wave $\left(\overline{\mathrm{V}}_{1}, \overline{\mathrm{I}}_{1}\right.$ ) on the impedance $\mathrm{Z}_{0}$ ) is a function of the beam loading which changes the apparent load at the end of the transmission line. The reflected power (wave $\left(\bar{V}_{2}, \bar{I}_{2}\right)$ on the impedance $Z_{0}$ ) is dissipated in the load. The equivalent circuit is shown in figure 17, where the generator is represented as a current source matched to the line impedance $Z_{0}$ (current $2 \vec{I}_{1}$ and shunt 
impedance $Z_{0}$ ) and driving the cavity impedance $Z_{c a v}$. The beam current $T_{B}$ is also injected into $Z_{\text {car }}$ and $Z_{0}$.

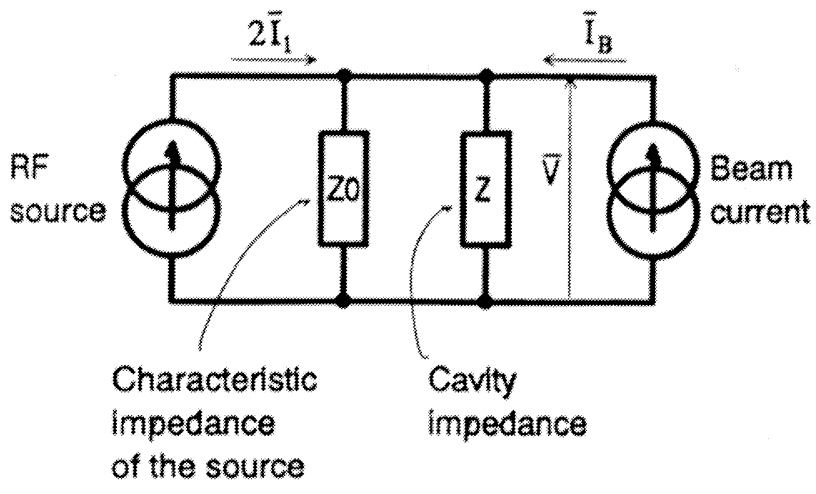

Figure 17 : Equivalent circuit

$$
\text { From figure } 17 \text { we derive : } 2 \overrightarrow{\mathrm{I}}_{1}=\overline{\mathrm{V}}\left(\frac{1}{\mathrm{Z}_{0}}+\frac{1}{\mathrm{Z}}\right)-\overrightarrow{\mathrm{I}}_{\mathrm{B}} \text {. }
$$

By definition the voltage $\bar{\nabla}_{2}$ of the reflected wave at the cavity is given by : $\bar{\nabla}_{2}=\bar{\nabla} \cdot \bar{\nabla}_{1}$.

Dividing by $Z_{0}$ and multiplying by 2 we get : $2 \overline{\mathrm{I}}_{2}=2 \frac{\bar{V}}{Z_{0}}-2 \overline{\mathrm{I}}_{1}$,

From (45) and (46) we deduce : $\quad 2 \overline{\mathrm{I}}_{2}=\overline{\mathrm{V}}\left(\frac{1}{\mathrm{Z}_{0}}-\frac{1}{\mathrm{Z}}\right)+\overline{\mathrm{I}}_{\mathrm{B}}$.

The reflected wave is generated at the cavity input port by a current source matched to the line impedance $Z_{0}$ (current $2 I_{2}$ and shunt impedance $Z_{0}$ ), and is propagating backwards to the load through the isolator.

\section{Steady-state operation}

With an ideal tuning system $\bar{V}$ is kept in phase with $\overline{\mathrm{I}}_{1}$. Consequently : 


$$
\begin{aligned}
& 2 l_{1}=v\left(\frac{1}{Z_{0}}+\frac{1}{R}\right)+I_{B} \sin \varphi_{\mathrm{B}} \\
& 2 l_{2}=v\left(\frac{1}{Z_{0}}-\frac{1}{R}\right)-I_{B} \sin \varphi_{B} .
\end{aligned}
$$

Let us select the critical coupling for the cavity $\left(Z_{0}=R\right)$ to minimize the RF power during storage $\left(\varphi_{\mathrm{B}}=0\right)$. The incident RF power being :

$$
P_{\text {incident }}=\frac{1}{2} Z_{0} I_{1}^{2}
$$

we get : $\quad P_{\text {incident }}=\frac{1}{2} \frac{V^{2}}{R}+\frac{1}{2} V I_{B} \sin \varphi_{B}+\frac{1}{2} Z_{0}\left(\frac{l_{B} \sin \varphi_{B}}{2}\right)^{2}$.

The first two terms in (49) correspond to the minimum power required to compensate for the cavity losses and to accelerate the beam. The third term is due to the mismatch in* duced by the beam loading (even though the tuning is perfect). For supra-conducting cavities, where $Z_{0}$ is very large, that power loss becomes important so that the coupling coefficient $\left(Z_{0} R\right)$ is rather selected to cancel the reflected power during beam acceleration [21].

\section{Transient analysis}

For a step-like current perturbation $\overline{\Delta \mathrm{I}_{\mathrm{B}}}$ in the beam current $\overline{\mathrm{I}}_{\mathrm{B}}$ the situation is the same as in section 6.1 . The amplifier must deliver $\bar{I}_{10}-\widetilde{U}_{B}$ in the cavity, as long as the tuning loop has not settled again at $\varphi_{\mathrm{L}}=0$. Assuming that critical coupling is used (room temperature cavity : $\left.\mathrm{Z}_{0}=\mathrm{R}\right)$, the incident power required can be shown to be :

$$
\hat{\mathrm{P}}_{\text {incident }}=\mathrm{P}_{0}\left(1+\left(\frac{\mathrm{V} \Delta \mathrm{I}_{\mathrm{B}}}{4 \mathrm{P}_{0}}\right)^{2}\right)
$$

where $P_{0}$ is the power needed to get $V$ when no acceleration takes place :

$$
P_{0}=\frac{1}{2} \frac{V^{2}}{Z_{0}}
$$


In the case of periodic transient beam loading, with $\tau<<$ TEV : $\Delta \mathrm{T}_{\mathrm{B}}=\hat{\mathrm{l}}_{\mathrm{B}}$ (local beam current). Consequently the peak RF power required is:

$$
\hat{\mathrm{p}}_{\text {incident }}=\mathrm{p}_{0}\left(1+\left(\frac{\mathrm{v} \hat{\mathrm{n}}_{\mathrm{B}}}{4 \mathrm{P}_{0}}\right)^{2}\right)
$$

which is minimum for: $\quad P_{0}=\frac{V \hat{I}_{B}}{4}$.

The minimum peak RF power is then : $\hat{\mathrm{p}}_{\text {incident }}=\frac{\mathrm{VI}}{2}$.

which depends on the local beam current $\hat{l}_{B}$.

\section{REFERENCES}

[1] F.J. Sacherer, "Bunch lengthening and microwave instability", IEEE Trans. Nuc. Sci., NS-24,1977, p. 1393

[2] D. Boussard, "Beam loading", 1985 CERN Accelerator School, Oxford (G.B.), CERN 8703, and CERN SPS/86-10 (ARF)

[3] D. Boussard, "Control of cavities with high beam loading", IEEE Trans. Nuc. Sci, NS* 32,1985, p. 1852

[4] F. Pedersen, "Beam loading effects in the CERN PS Booster", IEEE Trans. Nuc. Sci NS $22,1975, p .1906$

[5] K.W. Robinson, "Stability of beams in Radiofrequency systems", CEAL-1010, Feb. 1964

[6] A. Hoffman, "Coherent beam instabilities", these proceedings

[7] P.B. Wilson, "Transient beam loading in electron-positron storage rings", PEP Note-276

[8] G. Gelato et al., "Evolution of the RF system of the CPS Booster since the beginning of its operation", IEEE Trans. Nuc. Sci, NS-22, 1975, p. 1334

[9] D. Boussard, "Cavity compensation and beam loading instabilities", CERN/SPS/ARF Note $78-16$ 
(10) H. Frischholz, W. Schnell, "Compensation of beam loading in the ISR RF cavities", IEEE Trans. Nuc, Sci, NS-24, 1977, p. 1683

111) F. Ferger, W. Schnell, "The high power part of the RF system for the Intersecting Storage Rings (ISR)", 2nd Natl. Conf. on Part. Acc, Moscow, Sept. 1970

112) R. Garoby et al." "RF system for high beam intensity acceleration in the CERN PS", Proc. of the 1989 IEEE Part. Acc. Conf., Chicago, March 20-23, p. 135

[13] F. Pedersen, F. Sacherer, "Theory and performance of the longitudinal active damping system for the CERN PS Booster", IEEE Trans. Nuc. Sci., NS-24,1977, p. 1396

[14] G. Schneider, "Group delay equalizer for improvement of longitudinal beam stability in the PSB", CERN PS/RF/Note 914

[15] D. Boussard, G, Lambert, "Reduction of the apparent impedance of wide band accelerating cavities by RF feedback*, IEEE Trans. Nuc. Sci., NS-30, 1983, p. 2239

[16] F. Blas, R. Garoby, "Design and operational results of a one tum delay feedback for beam loading compensation on the CERN PS ferrite cavities", to be published in the Proc. of the 1991 Part. Acc. Conf, San Francisco, May 6-9

117 J. M. Baillod et al. "A second harmonic RF system with feedback-reduced gap impedance", IEEE Trans. Nuc. Sci., NS-30, 1983, p. 3499

[18] D. Boussard et al., "RF feedback applied to a multicell superconducting cavity", Proc. of the European Part. Acc. Conf., Rome, June 7-11, 1988, p. 985

[19] L.W. Funk et al, " $52 \mathrm{MHz}$ RF systems for PETRA II and HERA proton synchrotrons", Proc. of the 14th intern. conf. on high energy acc., Particle Accelerators, 1990, Vol.29, p.71

[20] S. Ninomiya et al." "The RF power system with beam loading cancellation for the KEKPS Booster", Proc. of the 14th intern. conf, on high energy acc., Particle Accelerators, 1990 , Vol.32, p. 69

[21] D. Boussard, "RF power estimates for a hadron collider", CERN SPS/ARF/Note 84-9 